Oral (007)

Gynecologic Cancer Screening

https://doi.org/10.3802/jgo.2021.32.S1.007

\section{Diagnosis of early-stage epithelial ovarian cancer (EOC) using comprehensive serum glycopeptide spectra analysis combined with artificial intelligence (CSGSA-AI)}

Masaru Hayashi, " Hiroaki Momose, Miwa Yasaka,

Hirofumi Kashiwagi, Hiroko Machida, Tetsuji lida, Masae Ikeda, Hiroshi Yoshida, Takeshi Hirasawa, Mikio Mikami

Tokai University, School of Medicine, Isehara, Japan

(hayashimasaru0620@hotmail.com)

Objective: We aimed to develop an artificial intelligence (AI)based comprehensive serum glycopeptide spectra analysis (CSGSA-AI) method in combination with convolutional neural network (CNN) to detect aberrant glycans in serum samples of patients with epithelium ovarian cancer (EOC).

Methods: A total of 97 serum samples were collected from patients with early-stage EOC at the time of ovarian mass detection prior to the initiation of any treatment (stage I). The non-EOC control group $(\mathrm{n}=254)$ comprised both healthy women $(n=220)$ and women with gynecologic benign diseases $(n=34)$. We used AlexNet, the latest CNN-based technology, as a discrimination tool for CSGSA to identify early-stage EOC. To facilitate CNN training, we converted numerical data of glycopeptide expression to 2D barcode images and let CNN learn and distinguish early-stage EOC. CNN was trained using 60\% samples and validated using $40 \%$ samples. To further enhance the learning efficacy and diagnostic performance of $\mathrm{CNN}$, we added cancer antigen 125 (CA125) and HE4 information into the 2D barcode by changing the color (multicolored model).

Results: The sensitivity, specificity, positive predictive value, negative predictive value and area under the curve (AUC) of CSGSAAI were $79 \%, 96 \%, 89 \%, 92 \%$, and $88 \%$, respectively. When CNN was trained with 2D barcodes colored on the basis of serum levels of CA125 and HE4 (multicolored model), AUC of 95\% was achieved. Conclusion: CSGSA-AI has the potential to be a useful tool for diagnosis of early-stage epithelial ovarian cancer.

Oral (008)

Epithelial Ovarian Cancer including Borderline Tumor https://doi.org/10.3802/jgo.2021.32.S1.008

\section{Quality-adjusted time without symptom or} toxicity and quality-adjusted progression-free survival of first-line maintenance niraparib in patients with advanced ovarian cancer
Pilar Barretina-Ginesta, ${ }^{1, *}$ Bradley J. Monk, ${ }^{2}$ Sileny Han, ${ }^{3}$ Bhavana Pothuri, ${ }^{4}$ Annika Auranen, ${ }^{5}$ Dana M. Chase, ${ }^{2}$ Domenica Lorusso, ${ }^{6}$ Charles Anderson, ${ }^{7}$ Sophie Abadie-Lacourtoisie, ${ }^{8}$ Noelle Cloven, ${ }^{9}$ Elena I. Braicu, ${ }^{10}$ Amnon Amit, ${ }^{11}$ Andrés Redondo, ${ }^{12}$ Ruchit Shah, ${ }^{13}$ Nehemiah Kebede, ${ }^{13}$ Carol Hawkes, ${ }^{14}$ Divya Gupta, ${ }^{15}$ Tatia Woodward, ${ }^{16}$ David M. O'Malley, ${ }^{17}$ Antonio González-Martín ${ }^{18}$ for the PRIMA/ENGOT-OV26/GOG-3012 Investigators

'Spanish Research Group for Ovarian Cancer (GEICO) and Medical Oncology Department, Institut Català d'Oncologia, Girona, Spain (mariapilar.barretina@udg.edu)

${ }^{2}$ Gynecologic Oncology Group (GOG) and Arizona Oncology (US Oncology Network), University of Arizona, Creighton University, Phoenix, AZ, USA ${ }^{3}$ Belgium and Luxembourg Gynaecological Oncology Group (BGOG) and Department of Gynaecology and Obstetrics, University Hospitals Leuven, Leuven, Belgium

${ }^{4}$ Gynecologic Oncology Group (GOG) and Department of Obstetrics and Gynecology, Laura Isaac Perlmutter Cancer Center, NYU Langone Health, New York, NY, USA

${ }^{5}$ Nordic Society of Gynaecological Oncology (NSGO) and Department of Obstetrics and Gynecology, Tampere University Hospital, Tampere, Finland

${ }^{6}$ Multicenter Italian Trial in Ovarian Cancer (MITO) and Fondazione IRCCS Istituto Nazionale Tumori, Milan, Italy

${ }^{7}$ Gynecologic Oncology Group (GOG) and Willamette Valley Cancer Institute, Eugene, OR, USA

${ }^{8}$ Spanish Research Group for Ovarian Cancer (GINECO) and Oncologie Médicale Gynécologique, Institut de Cancérologie de l'Ouest (ICO) - Site Paul Papin, Angers, France

${ }^{9}$ Gynecologic Oncology Group (GOG) and Division of Gynecologic Oncology, Texas Oncology (US Oncology Network), Fort Worth, TX, USA

${ }^{10}$ Arbeitsgemeinschaft Gynäkologische Onkologie (AGO) and Department for Gynaecology, Campus Virchow Clinic, Charité-Universitätsmedizin Berlin, Berlin, Germany

"Irish Society of Gynaecological Oncology (ISGO) and Division of Obstetrics and Gynecology, Rambam Medical Centre, Haifa, Israel ${ }^{12}$ Spanish Research Group for Ovarian Cancer (GEICO) and Medical Oncology, Hospital Universitario La Paz, Madrid, Spain

${ }^{13}$ Open Health Evidence and Access, Bethesda, MD, USA

${ }^{14} \mathrm{GlaxoSmithKline,} \mathrm{London,} \mathrm{UK}$

${ }^{15} \mathrm{GlaxoSmithKline,} \mathrm{Waltham,} \mathrm{MA,} \mathrm{USA}$

${ }^{16}$ GlaxoSmithKline, Philadelphia, PA, USA

${ }^{17}$ Gynecologic Oncology Group (GOG) and Division of Gynecologic Oncology, Ohio State University Comprehensive Cancer Center - James Cancer Hospital and Solove Research Institute (OSUCCC- James), Columbus, OH, USA

${ }^{18}$ Spanish Research Group for Ovarian Cancer (GEICO) and Medical Oncology Department, Clínica Universidad de Navarra, Madrid, Spain

Objective: This post hoc analysis of the phase 3 PRIMA trial examined quality-adjusted time without symptom or toxicity (QA-TWiST) and quality-adjusted progression-free survival (QAPFS) of patients with ovarian cancer on first-line maintenance niraparib vs. placebo.

Methods: QA-TWiST analyses were performed, defining TOX as the mean duration where patients experienced grade $\geq 2$ adverse events (fatigue/asthenia, nausea, vomiting, abdominal pain, and abdominal bloating) from randomization to disease progression/censoring. Mean QA-TWiST was calculated as 